JOURNAL DE PHYSIQUE

Colloque $\mathrm{C} 10$, supplément au $\mathrm{n}^{\circ} 12$, Tome 46 , décembre 1985 page Cl0-83

\title{
RESISTANCE STUDIES OF HYDROGEN DIFFUSION IN NIOBIUM
}

\author{
E.A. ClARK, P. TZANETAKIS* AND H.K. BIRNBAUM \\ Department of Metallurgy and Mining Engineering and Materials \\ Research Laboratory. University of Illinois, Urbana, \\ IL 61801, U.S.A.
}

\begin{abstract}
Hydrogen diffusion in $\mathrm{Nb}$ was studied using thermotransport to establish concentration gradients in a periodic width modulated sample. The resistance of the sample was measured as a function of time, by a Kelvin bridge circuit, as the perturbed hydrogen distribution returned to uniform concentration. Results for a $H / N b=0.001$ sample, measured in the temperature range $300 \mathrm{~K}$ and $180 \mathrm{~K}$ show the diffusivity of $\mathrm{H}$ to be two to four times larger than that measured by the Gorsky effect. When fitted to the Arrhenius relation, $Q=0.078 \pm .001 \mathrm{eV} /$ atom and $D_{0}=3.0 \pm 0.7 \times 10^{-4}$ $\mathrm{cm}^{2} / \mathrm{s}$.
\end{abstract}

\section{INTRODUCTION}

The diffusivity of hydrogen and its isotopes in $\mathrm{Nb}$ has been extensively studied using the Gorsky effect; a strain relaxation technique $/ 1 /$. Results for the solid solution a phase at low concentrations indicate a change of slope in the Arrhenius plot $(\log D$ vs. $1 / T)$, at about $250 \mathrm{~K}$, for hydrogen, but not for deuterium or tritium. Also, hydrogen shows a significantly smaller activation enthalpy of diffusion (both below and above $250 \mathrm{~K}$ ) than either deuterium or tritium $/ 2 /$. Efforts to apply classical or quantum theories of diffusion to these results are unsatisfactory $/ 3 /$. This report describes results of aiternative method to the Gorsky effect: using thermotransport to create a concentration gradient, instead of stress. In this technique, the relaxation of the resistance of a periodically width modulated sample is used as a measure of the hydrogen diffusivity. \footnotetext{
*Present address : Department of Physics, University of Crete,
Heraklion, Crete, Greece.
} 
EXPERIMENTAL

After outgassing $25 \mu \mathrm{m}$ thick $\mathrm{Nb}$ foil to $2 \times 10^{-8} \mathrm{Torr}$ and $2500 \mathrm{~K}$, the samples were shaped using photoresist techniques and electrochemical etching to produce a specimen shown in Fig. 1. The samples were used in pairs with one, used as a reference, having $\mathrm{H} / \mathrm{Nb}=0$. The other was charged with low levels of hydrogen using a coulombically titrated cathodic charging method (which avoids oxygen and nitrogen contamination). The hydrogen concentration was measured by the resistance increment. The specimens were clamped to a temperature controlled copper block.

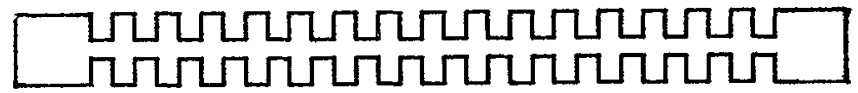

Fig. 1 - Drawing of a width modulated specimen.

The detection circuit is based on a double Kelvin bridge, which was balanced so that only the difference in resistance between the sample and reference was measured 14\%. Passing a $600 \mathrm{~mA}\left(6000 \mathrm{~A} / \mathrm{cm}^{2}\right)$ current through the sample and reference caused preferential heating of the thin parts, causing hydrogen thermotransport from the thin to the thick parts of the specimen. After the high current was removed, the bridge voltage was monitored as a function of time by a lock-in amplifier. This voltage is directly related to the difference between the concentration in the thin part $C_{1}$ and the average concentration $C_{0}$ by

$$
\frac{C_{1}-C_{0}}{C_{0}}=\frac{V_{k} \cdot(A+B)}{C_{0} \cdot k_{s} \cdot \rho_{H}^{0} \cdot I \cdot B} \cdot\left(\frac{d_{1}}{d_{2}}-1\right)^{-1}
$$

where $A$ and $B$ are bridge resistors $\left(6000 \Omega, V_{k}\right.$ is the bridge voltage, $k_{s}$ is the sample geometrical factor $\left(2 \times 10^{4} \mathrm{~cm}^{-1}\right) \rho_{0}^{0}$ is the resistivity per unit concentration of hydrogen $\left(0.64 \times 10^{-6} \Omega-\mathrm{Cm} / \mathrm{at} \%\right)$, I is the measuring current (50 $\mathrm{mA}$ ) and $d_{1}$ and $d_{2}$ are the thin and thick widths of the sample $(0.4 \mathrm{~mm}$ and $1.6 \mathrm{~mm}$, respectively).

For the results reported herein, at the start of the diffusion relaxation $V_{k}=$ $2.5 \times 10^{-7} \mathrm{~V}$, and $\mathrm{C}_{\mathrm{O}}=.0 .001 \mathrm{H} / \mathrm{Nb}$ so

$$
\left(c_{1}-c_{0}\right) / c_{0}=-0.01 \text {. }
$$

The heat of transport of $\mathrm{H}$ in $\mathrm{Nb}$ is $0.123 \mathrm{eV} /$ atom $/ 5,6 /$, and the effective temperature rise of the thin areas is estimated to be about $1 \mathrm{~K}$.

\section{RESULTS AND DISCUSSION}

A typical relaxation curve is shown in Fig. 2. The data were fitted using a method of non-linear least squares to several relaxation functions; a single exponential, a single exponential plus a linear ramp, and a sum of two exponentials (double exponentia1). The function chosen to be the best fit was determined by the minimal residual sum of squares and by inspection of the plots of the fitted function minus data point (residual) versus time. The double exponential was generally observed to provide the best fit to the data at the higher temperatures with the short, temperature independent relaxation time being associated with the thermal relaxation of the specimen after the heating current was turned off. The longer, temperature dependent relaxation was associated with hydrogen diffusion. At the lowest temperatures the thermal relaxation was best described by the fits of the single exponential with a smatl linear ramp function. In all cases, the hydrogen diffusion relaxation is described very well by a single exponential relaxation.

Fig. 3 is an Arrhenius plot of the hydrogen diffusivity for a sample with 0.1 at\% $H$. The diffusivity is calculated from the relaxation time $\tau$ by

$$
D=\frac{\lambda^{2}}{\pi^{2} \tau}
$$




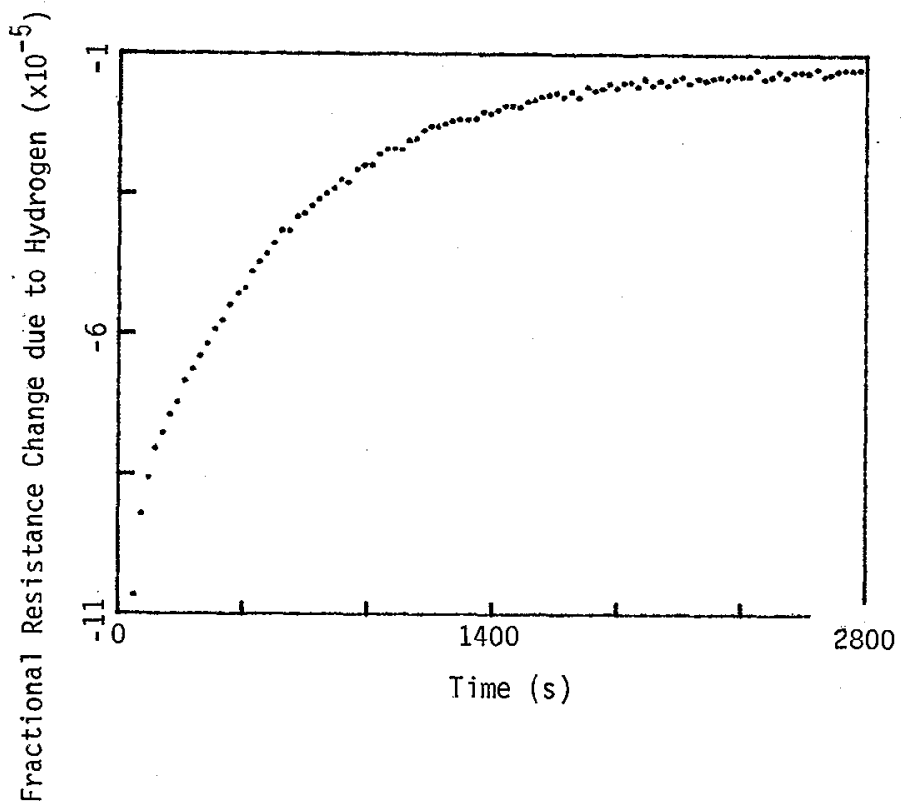

Fig. 2 - Relaxation of sample resistance vs. time due to $H$ diffusion at $220 \mathrm{~K}$ for $0.001 \mathrm{H} / \mathrm{Nb}$.

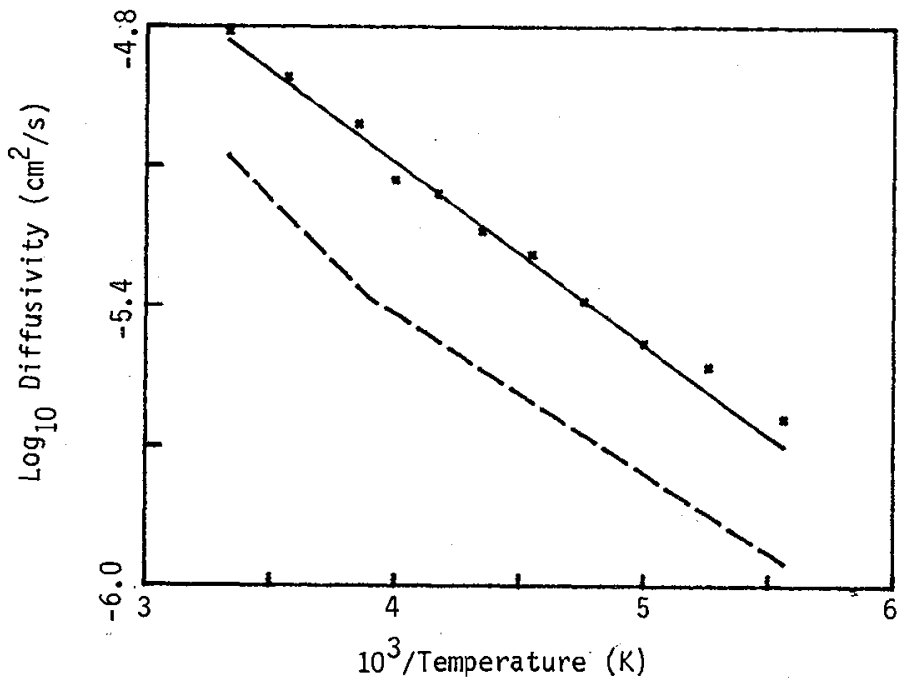

Fig. 3 - Log diffusivity vs. reciprocal temperature for $0.001 \mathrm{H} / \mathrm{Nb}$. $\mathrm{x}=$ data, solid line = Arrhenius fit to data, broken line = Gorsky effect.

where $\lambda$ is the wavelength of the width modulated sample $(1.7 \mathrm{~mm})$. Solution of the diffusion equation with the appropriate periodic boundary conditions for the width modulated sample can be shown to be a series of exponential relaxations. The behavior is dominated by the first term in the series which has the longest 
relaxation time and the largest amplitude. The dominant relaxation time is described by Eq. 1 which allows determination of diffusivity from the experimental data.

A7 so shown in Fig. 3 is the best fit of Volkl and Alefeld to the Gorsky effect data, showing the change of slope at $250 \mathrm{k} / 1 \%$. The present results are consistently above the Gorsky effect results (by a factor of two at $300 \mathrm{~K}$ and a factor of 4 at $180 \mathrm{~K}$ ). Correction of the present results for the mean field interaction of $\mathrm{H}$ would increase the diffusivity by $10 \%$. The results of a very similar thermotransport experiment by Wipf and Alefeld /5/ gave values of hydrogen diffusivity slightly less than the Gorsky effect and support the change of slope at about $250 \mathrm{~K}$. However, since these previous experiments used only one thin section (instead of the 16 used here), the relaxation curves were not single exponential and obtaining the relaxation times using a more complex fitting procedure than that used here. Differences between the present results and those previously reported may also stem from differences in purities of the $\mathrm{Nb}$. However, it must be pointed out that impurity trapping would result in a decrease of diffusivity and an increase in the activation enthalpy.

The present data were fitted to a single Arrhenius curve and to two Arrhenius curves. All the fitted parameters are presented in Table 1. Within the experimental error, the choice between the single Arrhenius or the fit to the two Arrhenius temperature dependences of the present data is unclear. Understanding the relationship of the present results to the previous Gorsky effect results will require additional experimental data.

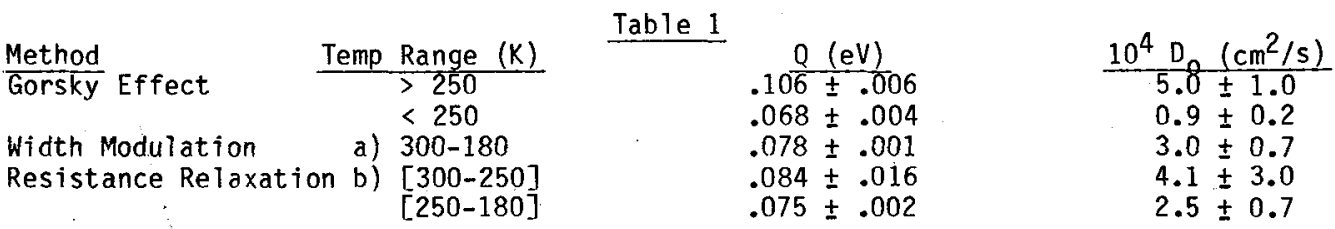

CONCLUSIONS

The diffusivity of hydrogen can be measured using a resistivity relaxation technique in which thermotransport of hydrogen in a width modulated specimen is used to perturb an initial uniform hydrogen distribution. The periodic form of the sample allows a simple relation between measured relaxation time and diffusivity to exist with the relaxation curves showing the expected single exponential behavior. Values of hydrogen diffusivity in a $\mathrm{H} / \mathrm{Nb}=0.001$ sample, between $180 \mathrm{~K}$ and $300 \mathrm{~K}$, are a factor of two $(300 \mathrm{~K})$ to four $(180 \mathrm{~K})$ higher than previous measurements obtained using the Gorsky effect. The present data do not support the existence of a change of slope of the Arrhenius plot of the hydrogen diffusivity as was previously reported at about $250 \mathrm{~K}$.

\section{ACKNOWLEDGEMENTS}

This work was supported by D.0.E. Office of Basic Energy Sciences under contract DE-AC02-76ER01198.

\section{REFERENCES}

/1/ J. Völk1 and G. Alefeld, chap. 5 in "Diffusion in Solids: Recent Developments", ed. A.S. Nowick, J.J. Burton, Academic Press, New York (1975).

121 K.W. Kehr, in "Electronic Structure and Properties of Hydrogen in Metals", NATO Conference Series VI: 6, ed. P. Jena, C.B. Sattherthwaite, PTenum, New York (1983). /3/ Zh Qi, J. Völk1, R. Lasser and H. Wenz1, J. Phys. F: Met. Phys. 13, 2053 (1983).

141 P. Tzanetakis and H.K. Birnbaum, Rev. Sci. Inst. 50, 672 (1979).

15/ H. Wipf and G. Alefeld, Phys. Stat. Sol. 23a, 175 (1975).

16/ D.T. Peterson and M.F. Smith, Met. Trans. 13A, 821 (1982). 